\title{
Reduced cancer risk in vegetarians: an analysis of recent reports
}

\author{
This article was published in the following Dove Press journal: \\ Cancer Management and Research \\ 20 December 2010 \\ Number of times this article has been viewed
}

\author{
Amy Joy Lanou' \\ Barbara Svenson ${ }^{2}$ \\ 'Department of Health and Wellness, \\ ${ }^{2}$ Ramsey Library, University of North \\ Carolina Asheville, Asheville, NC, USA
}

Correspondence: Amy Joy Lanou Department of Health and Wellness, CPO\# 2730, University of North Carolina Asheville, One University Heights, Asheville, North Carolina 28804, USA

$\mathrm{Tel}+\mathrm{I} 8282502317$

Fax + I 8282503856

Email alanou@unca.edu

\begin{abstract}
This report reviews current evidence regarding the relationship between vegetarian eating patterns and cancer risk. Although plant-based diets including vegetarian and vegan diets are generally considered to be cancer protective, very few studies have directly addressed this question. Most large prospective observational studies show that vegetarian diets are at least modestly cancer protective $(10 \%-12 \%$ reduction in overall cancer risk) although results for specific cancers are less clear. No long-term randomized clinical trials have been conducted to address this relationship. However, a broad body of evidence links specific plant foods such as fruits and vegetables, plant constituents such as fiber, antioxidants and other phytochemicals, and achieving and maintaining a healthy weight to reduced risk of cancer diagnosis and recurrence. Also, research links the consumption of meat, especially red and processed meats, to increased risk of several types of cancer. Vegetarian and vegan diets increase beneficial plant foods and plant constituents, eliminate the intake of red and processed meat, and aid in achieving and maintaining a healthy weight. The direct and indirect evidence taken together suggests that vegetarian diets are a useful strategy for reducing risk of cancer.
\end{abstract}

Keywords: diet, vegan, prevention

\section{Introduction}

Despite widespread research efforts and increasing treatment options, cancer remains a leading cause of death worldwide. In 2004, cancer accounted for $13 \%$ of deaths worldwide ( $\sim .4$ million people) with projections estimating an increase to 12 million deaths in $2030 .{ }^{1}$ Yet cancer is still considered a largely preventable disease with estimates of up to $90 \%-95 \%$ of the risk with roots in environment and lifestyle. ${ }^{2}$ Important lifestyle factors include tobacco use, diet, alcohol, sun exposure, environmental pollutants, infections, stress, obesity, and physical inactivity. Dietary factors were estimated to be responsible for $30 \%-35 \%$ of all cancers in the US in $1981 .{ }^{3}$ More recent estimates keep $35 \%$ as the overall impact of suboptimal dietary choices, but more specifically note that diet may be linked to as many as $70 \%$ of cases of colorectal and prostate cancer, $50 \%$ of cases for breast, endometrial, pancreatic, and gallbladder cancers, but only to about $20 \%$ of cases of lung, bladder, mouth, and esophageal cancers. ${ }^{4}$

While it is clear that dietary patterns and choices are important modulators of cancer risk, the question remains just what dietary pattern is optimal for primary prevention of cancer. The purpose, then, of this report is to review current evidence regarding the relationship between vegetarian eating patterns and cancer risk. Vegetarian eating patterns are defined as those that exclude all meats (beef, pork, lamb, poultry, fish, etc). Some vegetarians also exclude eggs but not dairy products, lacto vegetarians, some submit your manuscript $\mid$ www.dovepress.com

Dovepress

DOI: $10.2147 /$ CMR.S6910
Cancer Management and Research 201 I:3 I-8

(C) 201 I Lanou and Svenson, publisher and licensee Dove Medical Press Ltd. This is an Open Access article which permits unrestricted noncommercial use, provided the original work is properly cited. 
exclude dairy products but not eggs, ovo vegetarians, and some exclude all foods from animal sources (all meats, dairy, eggs, and other animal derived foods including gelatin, honey, casein, lactose, etc), vegans. Many individuals worldwide for cultural or socio economic reasons consume diets that contain very small to modest amounts of meat from any source. Some individuals may exclude red meat or some but not all types of meat (for example fish). While these diets are clearly based on plant foods, and therefore often deemed "plant-based diets", they are not vegetarian diets. Much of the evidence used to support the use of vegetarian diets for cancer risk reduction come from populations consuming plant-based diets and from studies that identify beneficial effects of specific foods or nutrients from plant sources. A brief overview of this literature is provided and then compared with the findings of studies specifically addressing the question of whether vegetarian dietary patterns reduce cancer risk.

\section{Plant-based dietary patterns and cancer risk}

Epidemiologic evidence from the Cornell-Oxford China Study conducted in the 1970s and 1980s demonstrated important relationships between dietary patterns and cancer risk and highlighted the importance of diets rich in whole plant foods for cancer prevention. ${ }^{5}$ The magnitude of difference in cancer risk within China ranges by more than a factor of 10 across the 65 counties studied. Campbell and colleagues found that a group of diseases (notably cancers of the colon, lung, breast, brain, as well as leukemia, cardiovascular disease, diabetes) were all associated with a diet of nutritional extravagance - meaning a diet that was associated with higher levels of blood cholesterol and blood urea nitrogen. These risk markers were directly associated with the intake of milk, meat, eggs, dietary fat, and animal protein and inversely associated with dietary fiber and legumes. In addition, breast cancer mortality increased with increasing dietary fat concentration and blood cholesterol levels. Higher blood levels of vitamin $\mathrm{C}$ and beta carotene, antioxidants provided by plant foods, were associated with lower rates of several cancers. ${ }^{5}$ In another report, Campbell and Chen make the strong statement that "there appears to be no threshold of plant food enrichment or minimization of fat intake beyond which further disease prevention does not occur". And they add that in the context of diets in China the addition of small amounts of foods from animal sources is associated with increased risk of chronic degenerative diseases including cancer. ${ }^{6}$
Similarly, Carroll and colleagues observed a strong relationship between animal fat intake and breast cancer mortality across 38 countries and no relationship between plant fat intake and breast cancer in these same countries. ${ }^{7}$ And in China during this time, where the variations in fat intake were mostly from animal-based foods and ranged from 6\%-24\% percent (all within "low-fat" ranges by US standards), breast cancer risk increased as fat intake increased. ${ }^{8}$

Evidence from migration studies in the 1980s also pointed to the hypothesis that plant-based dietary patterns are more cancer protective than standard Western dietary patterns that tended to be higher in animal food, sugar, and highly processed food products. For example, in one study breast cancer incidence for Japanese women who migrated to Hawaii increased nearly 3 -fold in the first generation and increased to 5-fold higher in the second generation. Similarly, colorectal cancer incidence jumped 5-fold for first generation immigrants in this same study population while stomach cancer incidence dropped by about half. ${ }^{9}$ Other migrant studies demonstrate dramatic shifts in site-specific cancer incidence when groups of people migrate to countries with different dietary and other lifestyle patterns. ${ }^{10}$ Worldwide nutrition transitions in less developed countries continue to rapidly unfold and are linked to cancer largely through their direct or indirect effect on body weight. The key changes increasing body weight and thereby increasing the risk of cancer are foods from animal sources, caloric sweeteners, and highly energy dense beverages and foods. ${ }^{11}$ This evidence clearly points to the importance of environment, including food availability and dietary patterns, for cancer risk.

\section{Dietary factors and cancer risk}

Numerous reviews have been written in the last 5 years that address the relationship between dietary factors and cancer risk. ${ }^{2,12-21}$ A few have highlighted vegetarian or vegan diets and overall and/or specific cancer risks and generally conclude that more information is needed to better clarify the relationship between various vegetarian dietary patterns cancer risk. ${ }^{19-21}$ Fraser, for example, highlights the beneficial relationship between vegetarian diets and overall cancer risk, but notes that the relationship between colon cancer risk and vegetarian dietary patterns needs to be resolved. ${ }^{19}$

In addition, the overall diet and cancer literature has been carefully reviewed and assessed by a team of experts convened by the World Cancer Research Fund (WCRF) and the American Institute for Cancer Research (AICR). ${ }^{10}$ From these reviews and the broader body of literature on diet and cancer, a number of specific dietary factors are clear. Red and 
processed meats increase the risk of some types of cancer. Diets rich in plant foods decrease the risk of many types of cancer; specifically, beneficial effects have been shown for fiber, fruits, vegetables, legumes including soy foods, and whole grains. Obesity increases the risk of some types of cancer. Many plant constituents, some nutrients, and other nonnutriuent phytochemicals increase immune function. Each of these will be discussed below and each one points to the potential usefulness of vegetarian dietary patterns for reduction of cancer risk.

\section{Meat and cancer}

Higher levels of meat, especially red meat (eg, beef, pork, lamb) and processed meat (eg, bacon, hotdogs, luncheon meat, chicken nuggets, and other salted or cured meats) have been linked to a variety of cancers in a number of studies. The WCRF report indicates that there is convincing evidence linking red and processed meat intakes with colorectal cancer, and suggestive evidence linking red meat and esophageal, lung, pancreatic, and endometrial cancer, and processed meat with esophageal, lung, stomach, and prostate cancer. ${ }^{10} \mathrm{~A}$ 2008 expert review also adds breast, bladder, and oral cancer to the list. ${ }^{2}$ This link between red and processed meat and cancer is supported by a recent prospective study addressing the relationship between meat intake and mortality. In this study over half a million people aged 50 to 71 years from the National Institutes of Health - AARP Diet and Health Study - were followed for 10 years. ${ }^{22}$ During that time 47,976 male and 23,276 female deaths were recorded. The men and women in the highest quartile of red meat intake had elevated risks for overall mortality (men: hazard ratio (HR), $1.31 ; 95 \%$ confidence interval [CI]: 1.27-1.35; women: HR, 1.36; $95 \%$ CI: $1.30-1.43$ ) and for overall cancer mortality (men: HR, 1.22; 95\% CI: 1.16-1.29; women: HR, 1.20; 95\% CI: 1.12-1.30). Higher levels of processed meat intake were also associated with increased cancer mortality risk in both men (HR, 1.12; 95\% CI: 1.06-1.19) and women (HR, 1.11; 95\% CI: 1.04-1.19) compared with lower intake of processed meat. These authors noted a 6-fold difference in average red meat intake between the lowest and highest quartiles expressed as g/1000 kcals (eg, for women $9.1 \mathrm{~g} / 1000 \mathrm{kcal}$ to $65.9 \mathrm{~g} / \mathrm{kcal}){ }^{22}$

When meat is cooked at high temperatures through pan frying, grilling, or barbequing potential carcinogenic compounds, heterocyclic amines (HCAs), are formed..$^{23,24}$ These compounds and have been strongly linked with increased risk of cancer at a number of sites. ${ }^{25} \mathrm{~A}$ recent study of grilled chicken dishes from popular chain restaurants in
California, found that all 100 samples contained some $\mathrm{PhIP}$ (one common HCA). The authors concluded that "although risks to the public have not been precisely calculated, strategies to reduce exposure to these and other carcinogens are warranted". ${ }^{26}$ They also note that HCA formation in plantbased grillable foods such as veggie burgers or mushrooms is highly unlikely. ${ }^{26}$ By definition, vegetarian diets are meatfree. Even semi vegetarian diets are usually devoid of red and processed meats. Studies linking the consumption of red meat and the consumption of HCAs to cancer risk highlight the potential benefits of vegetarian eating styles to reduction of cancer risk.

The conclusions that meat is linked with increased risk of some types of cancer and diets rich in plant foods are thought to be protective was recently underscored by the recommendations of the previously mentioned expert report published by the World Cancer Research Fund International. It stated among other recommendations that people should "eat mostly foods of plant origin" and "limit intake of red meat and avoid processed meat" to reduce cancer risk. ${ }^{10}$

\section{Body weight and cancer}

According to Demark-Wahnefried and coauthors, "the strongest current evidence for primary prevention and for improving outcomes after a diagnosis of cancer relates to the achievement of a healthy weight". ${ }^{27}$ Convincing evidence links higher levels of body fatness to increased risk of cancers of the esophagus, pancreas, colorectum, breast (postmenopausally), endometrium, and kidney. ${ }^{10}$ Consistent evidence also indicates that overweight and obesity are significant risk factors for cancer recurrence and comorbidities including cardiovascular disease and diabetes among cancer survivors. ${ }^{27}$ Adopting a physically active lifestyle can help individuals achieve a healthy weight and has been found to reduce the risk of colon and breast cancer. ${ }^{27}$ Vegetarians on average weigh $3 \%-20 \%$ less and have lower rates of obesity than omnivores. ${ }^{28}$ In addition, short-term studies of low-fat vegetarian and vegan diets have been successfully utilized to reduce body weight. ${ }^{28}$ For these reasons, adopting a lowfat vegetarian diet and regular physical activity will likely move individuals to a healthy weight and therefore reduce cancer risk.

\section{Whole plant foods and cancer}

Literally hundreds of studies have been conducted that address the relationship of some type of plant foods or plant food constituents and cancer risk. A clear majority of these studies demonstrate that fruits, vegetables, legumes, whole 
grains, spices, and seeds as well as specific types of food within these categories including citrus fruit, tomatoes, cruciferous vegetables, soybeans, and wheat, for example, reduce the risk of one or more types of cancer. ${ }^{10}$ Research on individual dietary factors and overall cancer risk is less clear. A recent report from the very large ( $>500,000$ study participants) European Investigation Into Cancer (EPIC) group finds a statistically significant, albeit very small inverse association with fruits and vegetables and overall cancer risk (200 g/day increased intake of total fruits and vegetables: HR, 0.97. 95\% CI: $0.96-0.99) .{ }^{29}$ These authors caution against over interpreting these results when making dietary recommendations for cancer risk reduction because the magnitude of the effect is small. ${ }^{29}$ The complexity of factors impacting overall cancer risk, the heterogeneity of cancer etiology, and the limitations in our dietary methods and the variation in dietary patterns beyond the specific factor studied may be responsible in part for the lack of clarity regarding the relationship between overall cancer risk and specific dietary factors. In addition, specific constituents in these whole foods such as soluble fiber, carotenoids, indoles, isoflavones, among hundreds of others have been linked to protection against specific cancers. ${ }^{2,10,17}$ Vegetarian and vegan diets tend to be higher in these protective plant foods and plant constituents than omnivorous diets. ${ }^{15,20,21,30-32}$ Vegetarian and other diets built mainly from plant foods would also be expected to support higher immune function, largely because they tend to be richer in cancer protective phytochemicals. , $14,15,17^{-1}$

The extent to which a vegetarian diet is cancer protective likely depends on how rich the dietary pattern is in these protective whole plant foods. The dietary patterns of some Western vegetarians may have macronutrient and micronutrient patterns that are not all that different than the dietary patterns of their omnivorous peers. Wide homogeneity of vegetarian diets exists such that individuals choosing selfreported vegetarian diets may exclude only some types of meat to all animal products, may include very large or very small amounts of highly processed food, may include only raw foods, or may vary widely with respect to cheese and other dairy product consumption. ${ }^{30}$

\section{Vegetarian dietary patterns and overall cancer risk}

Relatively few prospective studies have investigated the relationship between vegetarian diets and cancer risk. Overall meat-free diets appear to be at least modestly cancer protective $\left(10 \%-12 \%\right.$ reduction in risk) in most, ${ }^{33-35}$ but not all reports, ${ }^{36}$ however the results for specific cancers are less clear. This lack of clarity may have to do with the heterogeneity of vegetarian diets ${ }^{19,30}$ as they may range greatly in the ratio of animal to plant food eaten, the quality of the food, the limitations of measures used to quantify dietary intake, as well as other lifestyle and environmental factors that may impact risk of cancer in the populations studied. For example, a semi vegetarian who replaces most servings of beef and pork with servings of chicken and fish will have a very different nutrient composition compared with a vegetarian who consumes cheese or eggs at most meals or a vegan who consumes only foods from plant sources. The overall level of protective plant nutrients will vary with the total amount of whole plant foods consumed. ${ }^{31,32}$ Further, large prospective studies of diet typically rely on a single or infrequent food frequency questionnaires (FFQ) to characterize an individual's dietary pattern over a number of years. Even if FFQs were a perfect measure of dietary patterns, which they are not, it is a huge assumption to think that how a person eats is stable over a period of years. ${ }^{29}$

The most convincing recent direct evidence that vegetarian diets are associated with reduced cancer risk comes from a report on prospective data collected from the Oxford Vegetarian Study and the European Prospective Investigation into Cancer and Nutrition-Oxford (EPIC-Oxford) cohort. ${ }^{33}$ In this analysis, 61,566 British men and women were separated into three dietary pattern groups on the basis of 4 questions on an intake questionnaire. The resultant groups were meat eaters $(32,403)$, fish eaters (nonmeat eaters who did eat fish; 8562), and vegetarians $(20,601)$. After an average of 12.2 years follow-up, 3350 had been diagnosed with cancer (2204 in meat eaters, 317 in fish eaters, and 829 in vegetarians). Interestingly, there are vegans in this cohort, but according to the authors "there are currently too few cancers [among vegans] to be informative". Overall relative risk (RR) for all cancers (at 20 sites or groups of sites) was $0.88(0.81-0.96)$ in vegetarians and $0.82(0.73-0.93)$ in fish eaters compared to meat eaters after adjustment for age, smoking, alcohol use, body mass index, physical activity level and in women, parity, and oral contraceptive use..$^{33}$

The 63,550 vegetarian and nonvegetarian men and women in the prospective EPIC-Oxford were shown to have overall lower cancer incidence rates than the general population of the United Kingdom (overall standardized incidence rate $=72 \%$ ). Within this health conscious study population the vegetarians had an incident rate ratio of 0.89 (95\% CI: 0.80-1.00) after adjustment for age, sex, and smoking compared with nonvegetarians for overall malignant neoplasms. ${ }^{35}$ 
In contrast to the aforementioned studies, an earlier report on pooled mortality from five prospective studies showed no large differences in cancer mortality or mortality from all causes between vegetarians and nonvegetarians. ${ }^{36}$ However, among 34,192 Californian Seventh-day Adventists, vegetarians had a RR for total mortality of 0.80 (95\% CI: $0.74-0.87)$ compared with those who ate any meat products. Overall cancer risk was not reported, but the association in an overall lower risk of mortality was in part due to a lower risk of colon and prostate cancer. ${ }^{34}$

\section{Vegetarian dietary patterns and risk of specific cancers}

In the study that combined data from two large British cohorts (EPIC-Oxford and the Oxford Vegetarian Study), the RR for cancer among vegetarians compared to meat eaters had significant heterogeneity and were strikingly lower at four sites: stomach cancer (RR, 0.36; 95\% CI: 0.16-0.78), bladder cancer (RR, 0.47; 95\% CI: 0.25-0.89), ovarian cancer (RR, 0.69; 95\% CI: 0.45-1.07), and cancers of the lymphatic and hematopoietic tissues (RR, 0.55; 95\% CI: $0.38-0.78) .{ }^{33}$ Based on a total of 50 incident cases of cervical cancer, vegetarians and fish eaters had a higher RR than meat eaters (2.08 and 2.05, respectively). Risk of cancers of the colorectum, upper gastrointestinal tract, pancreas, lung, skin (melanoma), endometrium, kidney, and brain were not significantly different among the diet groups. Prostate cancer risk was significantly lower among fish eaters than meat eaters (RR, 0.57; 95\% CI: 0.33-0.99) but not among vegetarians (RR, 0.87; 95\% CI: 0.64-1.18). ${ }^{33}$ In some cases even this large study may have been underpowered to detect a difference if people had changed eating patterns during the 12 years after the initial diet assignment or in cases where the total number of incident cancer cases was small. ${ }^{33}$ Nonetheless the study shows that overall cancer risk is roughly $12 \%$ lower among British vegetarians and fish eaters who avoid other types of meat compared to their meat eating peers, likely, in part, due to lower RR of stomach, bladder, ovarian, and lymphatic cancers. ${ }^{33}$

A report from the Oxford Vegetarian Study also did not find a statistically significant difference in the incidence of colorectal cancer among 10,998 men and women between vegetarians and meat-eaters $(0.85$; 95\% CI: 0.55-1.32). In this study 95 incident cases of colorectal cancer were recorded after 17 years of follow-up. ${ }^{37}$ In contrast, the colorectal cancer rate was higher among vegetarians than nonvegetarians (1.39; 95\% CI: 1.01, 1.91) in the EPIC-Oxford study. ${ }^{35}$ However, among a cohort of 34,192 Californian Seventh-day Adventists after 6 years of follow-up, the RR of colon cancer was 1.88 (1.24-2.87) in nonvegetarians compared with vegetarians indicating that the dietary factors important in colorectal cancer may be more complex than whether or not meat is included in the diet. ${ }^{34}$

Also in the study of Californian Seventh-day Adventists the RR of prostate cancer was $1.54(1.05-2.26)$ in nonvegetarians compared with vegetarians $(P<0.05) .{ }^{34}$ Risk of breast (RR, 1.25), lung (RR, 1.16), and uterine cancer (RR, 1.17) was not significantly different between diet groups in this population. Nonvegetarians consumed at least one serving of meat per week (on average $\sim 4.25$ servings of any type of meat, poultry, or fish/week) compared with no servings among the vegetarians. The dietary profiles between the groups differed in other ways as well. Nonvegetarians were more likely to drink coffee and alcohol and less likely to eat nuts, beans, and vegetarian meat substitutes than the

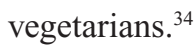

The UK Women's Cohort Study showed that vegetarians have a lower risk of breast cancer than meat eaters in pooled analysis of 10 cohort and case-control studies. For the cohort studies, the summary risk was 1.11 and for the case-control studies it was 1.57. The total risk looking at data from all studies analyzed was 1.24 ( $P$ for heterogeneity was 0.005$).{ }^{38}$ In another analysis of the EPICOxford study, researchers did not find a difference in RR of breast cancer in vegetarians compared with meat eaters (HR, 0.88; 95\% CI: $0.71-1.10 ; P=0.26) .{ }^{39}$

The lack of a significant relationship for vegetarian diets and colorectal cancer and prostate cancer in some studies is unexpected. The report from the WCRF/AICR in 2007 concluded that the evidence is convincing that high intakes of red meat and processed meat increase the risk of colorectal cancer. This same report states that limited evidence links processed meat as well as milk and dairy products with prostate cancer. ${ }^{10}$

\section{Discussion and implications}

Perhaps the most surprising outcome of reviews linking dietary factors and cancer prevention is how few strong conclusions can be drawn from the large number of high quality studies published on this topic. In the case of vegetarian diets and cancer, most research points to the potential viability of using vegetarian diets as a strategy for cancer prevention. And while the recent evidence from large prospective studies largely supports this strategy, the findings are neither as strongly supportive nor as clear as one would anticipate from the indirect evidence. 
One of the key limitations of research on vegetarian diets and cancer prevention is that randomized clinical trials investigating this relationship would be very costly and most possible designs would likely be unethical. This means that large, long-term, prospective trials are the gold standard of research investigating this question, but of course, these come with limitations. Although cancer is one of the leading causes of death worldwide, the incidence of new diagnosis for any specific type of cancer is low enough that large populations are required. In addition, the number of known and unknown factors that impact cancer development and prevention is large, varied, and likely to interact with each other. Some factors may even be protective for some types of cancer and causal or promoting for other types of cancer. For example, adequate vitamin D status is thought to be protective against prostate cancer, but sun exposure (the stimulus for vitamin $\mathrm{D}$ production in the human body) is a major risk factor for skin cancer. Similarly higher calcium intakes are associated with decreased risk of colon cancer but increased risk of prostate cancer.

Perhaps even more problematic for nutrition and cancer research generally and the question at hand specifically are the problems inherent in measuring food intake and quantifying dietary patterns. Given that in these "gold standard" studies dietary habits were measured using a food frequency questionnaire at the beginning of the study and then the participants are followed for 7 to 12 years with no additional measures of dietary patterns, ${ }^{33-35}$ it is remarkable that we are able to see any relationship between vegetarian diets and cancer risk. Nothing is known about the participant's food intake during those intervening years. Researchers don't even know whether the individuals continued to follow the same general dietary pattern that they did when they were classified into a dietary pattern category until their diagnosis or death. Many people change their dietary strategies over time making it likely to be more difficult to tease out associations between any given dietary pattern and cancer risk or mortality and more likely to dampen an association that is detectable.

Nonetheless, in large prospective cohort studies vegetarian diets have been shown to be at least modestly cancer protective (10\%-12\% reduction in risk) in most, ${ }^{33-35}$ but not all recent studies. ${ }^{36}$ Results for specific cancers are less clear although there is some observational evidence that vegetarian diets may reduce risk of prostate, ${ }^{34}$ breast, ${ }^{38}$ colon,,${ }^{34}$ stomach cancer, ${ }^{33}$ bladder cancer, ${ }^{33}$ ovarian cancer, ${ }^{33}$ and cancers of the lymphatic and hematopoietic tissues. ${ }^{33}$ Choosing a vegetarian dietary pattern is an easy way to follow the expert recommendations to "eat mostly foods of plant origin" and "limit intake of red meat and avoid processed meat" to reduce cancer risk. ${ }^{10}$

In addition, low-fat, whole foods vegan, vegetarian, or near vegetarian diets are often recommended as part of a strategy to treat or reduce the recurrence of cancer. ${ }^{40-44} \mathrm{~A}$ few published studies have investigated the use of vegetarian or vegan diets for cancer treatment or reduction of recurrence or as an adjunct to other cancer treatments. Because watchful waiting is one possible treatment option for early stage prostate cancer, clinical trials of diet and lifestyle change are more likely with this type of cancer than others. A one-year clinical trial of 93 men with biopsy-proven prostate cancer randomly assigned men who had chosen not to undergo any conventional treatment to an intensive lifestyle change group or to a usual care group. Participants in the intensive lifestyle changes group were asked to consume a low-fat vegan diet, do regular exercise and stress management, and to participate in support sessions. Prostate-specific antigen (PSA) decreased $4 \%$ in the intensive lifestyle change group but increased $6 \%$ in the control group $(P=0.016)$. None of the experimental patients, but six control patients, underwent conventional treatment due to a measurable progression of the prostate cancer. ${ }^{45}$ In a follow-up report from 2 years after the end of this trial, 13 of the 49 control patients and 2 of the 43 patients in the intensive lifestyle treatment had undergone conventional prostate cancer treatment $(P<0.05)$. The authors concluded that "patients with early-stage prostate cancer choosing active surveillance might be able to avoid or delay conventional treatment for at least 2 years by making changes in their diet and lifestyle". ${ }^{46}$ In a recent review of 8 observational and 17 clinical or laboratory trials of diet and survival after prostate cancer, predominantly plant-based diets that are high in fiber and phytonutrients and low in fat and saturated fat were determined to favorably influence outcomes for prostate cancer patients. ${ }^{41}$ Similar conclusions were drawn about the use of plant-based diets for survival of breast cancer in another recent review. ${ }^{42}$ Based on an exhaustive review of the diet and cancer literature, the World Cancer Research Fund makes the simple recommendation for cancer survivors to "follow the recommendations for cancer prevention" which as stated above include eating mostly plant foods and limiting red meat and avoiding processed meats ${ }^{6}$ which is clearly consistent with vegetarian dietary patterns.

Additional research is needed to clarify and strengthen our understanding of the usefulness of vegetarian dietary patterns for the prevention and treatment of cancer. Large prospective trials with repeated measurements of dietary intake are needed to clarify the role of dietary patterns in 
cancer prevention. Larger clinical trials studying the effectiveness of vegetarian diets and other dietary interventions on prevention in high risk individuals as well as trials that include vegetarian diets as a treatment or adjunct to treatment of cancer or prevention of recurrence will aid researchers in crafting dietary recommendations for prevention, treatment, and the prevention of cancer recurrence. Until then, dietitians, physicians, and other health care givers can safely recommend vegetarian and other diets built from whole foods from plant sources for cancer prevention as the literature is clear that vegetarian diets are likely to be protective and certainly do not increase cancer risk.

\section{Conclusion}

Although plant-based diets including vegetarian and vegan diets are generally considered to be cancer protective, surprisingly very few studies have directly addressed this question. However, a broad body of evidence links specific plant foods such as fruits and vegetables, plant constituents such as fiber, anti-oxidants and other phytochemicals, and achieving and maintaining a healthy weight to reduced risk of cancer diagnosis and recurrence. ${ }^{10,13}$ And, research links meat, especially red and processed meats, consumption to increased risk of several types of cancer. ${ }^{2,10,22}$ Vegetarian and vegan diets increase beneficial plant foods and plant constituents, ${ }^{16,20,21,30-32}$ eliminate the intake of red and processed meat by definition, and aid in achieving and maintaining a healthy weight. ${ }^{28}$ The few reports that have addressed whether vegetarian diets can be used for management or prevention of recurrence of cancer are positive. ${ }^{41,42,45,46}$ The direct and indirect evidence taken together suggests that vegetarian diets are a useful strategy for cancer prevention.

\section{Disclosure}

The authors report no conflicts of interest in this work.

\section{References}

1. World Health Organisation [WHO]. Projections of mortality and burden of disease, 2004-2030. Available from: http://www.who.int/healthinfo/ global_burden_disease/projections/en/index.html. Accessed 18 Aug 2010.

2. Anand P, Kunnumakkara AB, Sundaram C, et al. Cancer is a preventable disease that requires major lifestyle changes. Pharm Res. 2008;25(9):2097-2116.

3. Doll R, Peto R. The causes of cancer: quantitative estimates of avoidable risks of cancer in the United States today. $J$ Natl Cancer Inst. 1981;66:1191-1308.

4. Willett WC. Diet and cancer. Oncologist. 2000;5:393-404.

5. Campbell TC, Parpia B, Chen J. Diet, lifestyle, and the etiology of coronary artery disease: The Cornell China study. Am J Cardiol. 1998;82:18T-21T.
6. Campbell TC, Chen J. Diet and chronic degenerative diseases: perspectives from China. Am J Clin Nutr. 1994;59:1153S-1161S.

7. Carroll KK, Braden LM, Bell JA, et al. Fat and cancer. Cancer 1986;58:1818-1825.

8. Campbell TC, Campbell TM. The China Study. Callas TX: BenBella Book; 2005:86-87.

9. Kolonel LN, Hinds MW, Hankin JH. Cancer patterns among migrant and native-born Japanese in Hawaii in relation to smoking, drinking and dietary habits. In: Gelboin HV, et al (editors). Genetic and Environmental Factors in Experimental and Human Cancer. Tokyo: Japan Sci Soc Pr. 1980:327-340.

10. World Cancer Research Fund/American Institute for Cancer Research. Food, Nutrition, Physical Activity, and the Prevention of Cancer: a global perspective. Washington DC: AICR. 2007:22-25.

11. Popkin BM. Understanding global nutrition dynamics as a step towards controlling cancer incidence. Nature Rev Cancer. 2007;7:61-67.

12. Tyrovolas S, Panagiotakos DB. The role of Mediterranean type diet on the development of cancer and cardiovascular disease, in the elderly: a systematic review. J Maturitas. 2009;65(2):122-130.

13. Demark-Wahnefried W, Rock CL, Patrick K, Byers T. Lifestyle interventions to reduce cancer risk and improve outcomes. Am Fam Physician. 2008;77(11):1573-1580.

14. Russo GL. Ins and outs of dietary phytochemicals in cancer chemoprevention. Biochem Pharm. 2007;74:533-544.

15. Reilly JK. Diet and immune function in cancer prevention and survival. Hematol Oncol News Iss. 2006 Mar:30-33.

16. Divisi D, Di Tommaso S, Salvemini S, Garramone M, Crisci R. Diet and cancer. Acta Biomed. 2006:118-123.

17. Béliveau R, Gingras D. Role of nutrition in preventing cancer. Can Fam Physician. 2007;53:1905-1911.

18. Williams MT, Hord NG. The role of dietary factors in cancer prevention: beyond fruits and vegetables. Nutr Clin Prac. 2005;20:451-459.

19. Fraser GE. Vegetarian diets: what do we know of their effects on common chronic diseases? Am J Clin Nutr. 2009;89(Suppl):1607S-1612S.

20. Craig WJ. Health effects of vegan diets. Am J Clin Nutr. 2009: 89(Suppl):1627S-1633S.

21. Craig WJ, Mangels AR; American Dietetic Association. Position of the American Dietetic Association: vegetarian diets. J Am Diet Assoc. 2009;109:1266-1282.

22. Sinha R, Cross AJ, Graubard BI, Leitzmann MF, Schatzkin A. Meat intake and mortality: a prospective study of over a half a million people. Arch Intern Med. 2009;169(6):562-571.

23. Felton JS, Knize MG, Wu RW, et al. Mutagenic potency of food derived heterocyclic amines. Mut Res. 2007;616:90-94.

24. Knize MG, Felton JS. Formation and human risk of carcinogenic heterocyclic amines formed from natural precursors in meat. Nutr Rev. 2005;63(5):158-165.

25. Zheng W, Lee SA. Well-done meat intake, heterocyclic amine exposure, and cancer risk. Nutr Cancer. 2009;61(4):437-446.

26. Stoick KM, Erickson MA, Sandusky CB, Barnard ND. Detection of $\mathrm{PhIP}$ in grilled chicken entrees at popular chain restaurants throughout California. Nutr Cancer. 2008;60(5):1-9.

27. Demark-Wahnefried W, Rock CL, Patrick K, Byers T. Lifestyle interventions to reduce cancer risk and improve outcomes. Am Fam Phyiscian. 2008;77(11):1573-1580.

28. Berkow SE, Barnard N. Vegetarian diets and weight status. Nutr Rev. 2006:64(4):175-188.

29. Boffett P, Couto E, Wichmann J, Ferrari P, et al. Fruit and vegetable intake and overall cancer risk in the European Prospective Investigation Into Cancer and Nutrition (EPIC). J Natl Cancer Inst. 2010;102:1-9.

30. Leitzmann C. Vegetarian diets: what are the advantages? In: Elmadfa I, editor. Diet Diversification and Health Promotion. Forum Nutr. Basel: Karger; 2005, pp. 147-156.

31. Turner-McGrievy GM, Barnard ND, Cohen J, et al. Changes in nutrient intake and dietary quality among participants with type 2 diabetes following a low-fat vegan diet or a conventional diabetes diet for 22 weeks. J Am Diet Assoc. 2008;108:1636-1645. 
32. Dewell A, Weidner G, Sumner MD, Chi CS, Ornish D. A very-low fat vegan diet increases intake of protective dietary factors and decreases intake of pathogenic dietary factors. J Am Diet Assoc. 2008;108:347-356.

33. Key TJ, Appleby PH, Spencer EA, et al. Cancer incidence in British vegetarians. Br J Cancer. 2009;101:192-197.

34. Fraser GE. Associations between diet and cancer, ischemic heart disease, and all-cause mortality in non-Hispanic white California Seventh-day Adventists. Am J Clin Nutr. 1999;70(Suppl):532S-538S.

35. Key TJ, Appleby PH, Spencer EA, Travis RC, Roddam AW, Allen NE. Cancer incidence in vegetarians: results from the European Prospective Investigation in Cancer and Nutrition (EPIC-Oxford). Am J Clin Nutr. 2009;89(Suppl):1620S-1626S.

36. Key TJ, Fraser GE, Thorogood M, et al. Mortality in vegetarians and nonvegetarians: detailed findings from a collaborative analysis of 5 prospective studies. Am J Clin Nutr. 1999;70(Suppl): 516S-524S.

37. Sanjoaquin MA, Appleby PN, Thorogood M, JI Mann JI, Key TJ. Nutrition, lifestyle and colorectal cancer incidence: a prospective investigation of 10,998 vegetarians and non-vegetarians in the United Kingdom. Brit J Cancer. 2004;90:118-121.

38. Taylor VH, Misra M, Mukherjee SD. Is red meat intake a risk factor for breast cancer among premenopausal women? Breast Cancer Res Treat. 2009;117:1-8.
39. Travis RC, Allen NE, Appleby PN, Spencer EA, Roddam AW, Key TJ. A prospective study of vegetarianism and isoflavone intake in relation to breast cancer risk in British women. Int $J$ Cancer. 2008;122(3):705-710.

40. Freedland SJ, Aronson WJ. Dietary intervention strategies to modulate prostate cancer risk and prognosis. Curr Opin Urol. 2009;19:263-267.

41. Berkow SE, Barnard ND, Saxe GA, Ankerberg-Nobis T. Diet and survival after prostate cancer diagnosis. Nutr Rev. 2007;65(9):391-403.

42. Ferdowsian HR, Barnard ND. The role of diet in breast and prostate cancer survival. Ethnicity Dis. 2007;17 Suppl 2:18S-22S.

43. McCarty MF, Block KI. Toward a core nutraceutical program for cancer management. Integr Canc Ther. 2006:5(2):150-171.

44. Kushi LH, Cunningham JE, Hebert JR, et al. The macrobiotic diet in cancer. J Nutr. 2001;131:3056S-3064S.

45. Ornish D, Weidner G, Fair WR, et al. Intensive lifestyle changes may affect the progression of prostate cancer. $J$ Urol. 2005;174(3):1065-1069.

46. Frattaroli J, Weidner G, Dnistrian AM, et al. Clinical events in prostate cancer lifestyle trial: results from two years of follow-up. Urology. 2008;72(6):1319-1323.

\section{Publish your work in this journal}

Cancer Management and Research is an international, peer-reviewed open access journal focusing on cancer research and the optimal use of preventative and integrated treatment interventions to achieve improved outcomes, enhanced survival and quality of life for the cancer patient. The journal welcomes original research, clinical \& epidemiological

\section{Dovepress}

studies, reviews \& evaluations, guidelines, expert opinion \& commentary, case reports \& extended reports. The manuscript management system is completely online and includes a very quick and fair peerreview system, which is all easy to use. Visit http://www.dovepress.com/ testimonials.php to read real quotes from published authors. 